\title{
Differential diagnostic challenge of chronic neutrophilic leukemia in a patient with prolonged leukocytosis
}

\author{
Daniel Neureiter • Ralf Kemmerling • Matthias Ocker • \\ Christoph Seidlhofer • Viktoria Faber • \\ Markus Stöcher • Richard Greil • Otto Dietze
}

Received: 20 March 2008 / Accepted: 26 March 2008/Published online: 19 April 2008

(C) Springer-Verlag 2008

\begin{abstract}
Our interesting case deals with the clinical and morphological aspects of a chronic neutrophilic leukemia and the critical evaluation of differential diagnosis of leukemoid reaction in bone marrow biopsies.
\end{abstract}

Keywords Leukocytosis · Leukemoid reaction .

Chronic neutrophilia leukemia $\cdot$ Differential diagnosis

\section{Introduction}

Our interesting case deals with the clinical and morphological aspects of a chronic neutrophilic leukemia (CNL) and the critical evaluation of differential diagnosis of leukemoid reaction in bone marrow biopsies.

V. Faber $\cdot$ M. Stöcher $\cdot$ R. Greil

Medical Clinic III, Paracelsus Private Medical University,

Salzburger Landeskliniken,

Salzburg, Austria

D. Neureiter $\cdot$ R. Kemmerling $\cdot$ M. Ocker $\cdot$ O. Dietze Institute of Pathology, Paracelsus Private Medical University, Salzburger Landeskliniken,

Salzburg, Austria

C. Seidlhofer

Medical Clinic,

Hospital Barmherzige Brüder,

Salzburg, Austria

D. Neureiter $(\bowtie)$

Institute of Pathology,

Paracelsus Private Medical University,

Salzburger Landeskliniken,

Müllner Hauptstr. 48,

5020 Salzburg, Austria

e-mail: d.neureiter@salk.at

\section{Case}

An 81-year-old male patient was admitted to our hospital suffering from diabetes mellitus, chronic coronary heart disease, and Coombs-positive hemolysis as well as a suspicious war wound associated with recurrent osteomyelitis of the left femur. The indication for a bone marrow biopsy was a prolonged neutrophilic leukocytosis in combination with anemia and enhanced erythrocyte sedimentation rate (see Table 1). In 2004, the first bone marrow biopsies (without bone marrow smear) were sent from an external clinic to our institute. The bone marrow routine histology (see Fig. 1) displayed a prominent hyperplasia of hematopoiesis with striking dominance of granulopoiesis and hyoplasia of erythropoiesis without signs of dysplasia. The megakaryocytopoiesis showed normal number without significant signs of dysplasia. No relevant number of blasts or immature granulocytes was found. The age-related adipose tissue was significantly reduced and enhanced iron deposits in macrophages were observed (see Fig. 1). At this time a bone marrow smear was not carried out as these morphological findings were interpreted as reactive in circumstances of the clinically communicated osteomyelitis.

An additional outpatient laboratory analysis was performed in the year 2005 confirming persisting leukocytosis (see Table 1).

Because leukocytosis continued further, the patient was admitted to our clinic in 2006. At the admission, enhanced white blood cell count of $30 \mathrm{~g} / 1$ was observed rising up from 30 to $63.12 \mathrm{~g} / 1$ (the latter at the day of death) during the whole period of hospitalisation (from 9 September 2006 to 2 October 2006). Additionally, the relative percentage of band and segmented neutrophilic granulocytes was elevated at admission without evident monocytosis or immature cells (such as promyelocytes, myelocytes, or metamyelocytes), 
Table 1 Summary of the documented clinical and relevant laboratory findings during the last clinical stays

\begin{tabular}{|c|c|c|c|c|}
\hline & Normal range & 2004 & 2005 & 2006 \\
\hline Hepatosplenomegaly $^{\mathrm{a}}$ & & NA & NA & Yes \\
\hline Vit-B12 (pg/ml) & $>170$ & 310 & 313 & $>2000$ \\
\hline $\mathrm{CRP}[\mathrm{mg} / \mathrm{dl}]^{\mathrm{b}}$ & $<0.6$ & 1.5 & 1.5 & 2.1 \\
\hline Leukocytes $[\mathrm{g} / 1]^{\mathrm{b}}$ & $4-10$ & 14.0 & 11.8 & $30.7\left(33.9^{c}\right)$ \\
\hline Promyelocytes [\%] $]^{\mathrm{b}}$ & $<1$ & 0 & NA & $2\left(2^{c}\right)$ \\
\hline Myelocytes $[\%]^{\mathrm{b}}$ & $<1$ & 1 & NA & $3\left(5^{c}\right)$ \\
\hline Metamyelocyes [\%] $]^{\mathrm{b}}$ & $<1$ & 1 & NA & $1\left(7^{c}\right)$ \\
\hline Band granulocytes $[\%]^{\mathrm{b}}$ & $<3$ & 10 & NA & $10\left(9^{\mathrm{c}}\right)$ \\
\hline $\begin{array}{l}\text { Neutrophilic } \\
\text { granulocytes [\%] }\end{array}$ & $50-70$ & 59 & 70.8 & $65\left(57^{\mathrm{c}}\right)$ \\
\hline Lymphocytes $[\%]^{\mathrm{b}}$ & $20-40$ & 14 & 15.3 & $4\left(5^{c}\right)$ \\
\hline Monocytes [\%] ${ }^{\mathrm{b}}$ & $1-11$ & 4 & NA & $5\left(15^{\mathrm{c}}\right)$ \\
\hline Eosinophils [\%] ${ }^{\mathrm{b}}$ & $<4$ & 11 & NA & $9\left(0^{\mathrm{c}}\right)$ \\
\hline Basophils [\%] ${ }^{\mathrm{b}}$ & $<1$ & 0 & NA & $1\left(0^{c}\right)$ \\
\hline
\end{tabular}

NA Not available

${ }^{\mathrm{a}}$ By clinical or physical investigation

${ }^{\mathrm{b}}$ At admission

${ }^{\mathrm{c}} 5$ days before death

whereas it must be pointed out that the last differential blood smear was performed at the same time as the respiratory infection was detected being associated with a left shift (see Table 1). Other parameters of peripheral blood analysis were: $\mathrm{Hgb}, 8.2 \mathrm{~g} / \mathrm{dl}$; Hkt, 28.0\%; RBC, $2.6 \mathrm{~T} / \mathrm{l}$; MCV, $106.1 \mathrm{fl}$; MCH, $31.1 \mathrm{pg}$; and PLT, $45 \mathrm{~g} / \mathrm{l}$.

Interestingly, compared to the earlier clinical stays, Creactive protein (CRP) was continuously low and vitamin $\mathrm{B}_{12}$ was strongly elevated during the last stay (see Table 1). Most of the other laboratory findings were associated with the known diseases of the patient or elevated premortem, especially the kidney function ((electrolytes: chloride, $96 \mathrm{mmol} / \mathrm{l}$; sodium, $136 \mathrm{mmol} / \mathrm{l}$; potassium, $4.3 \mathrm{mmol} / \mathrm{l}$; calcium, $1.82 \mathrm{mmol} / \mathrm{l}$ ) (proteins: total protein/ serum, $6.7 \mathrm{~g} / \mathrm{dl}$ ) (liver function: GOT, $68 \mathrm{U} / 1$; GPT, $22 \mathrm{U} / \mathrm{l}$; gGT, $92 \mathrm{U} / \mathrm{l}$; cholinesterase, $4.2 \mathrm{U} / \mathrm{ml}$; alkaline phosphatase, $125 \mathrm{U} / \mathrm{l}$ ) (kidney function: creatinine up to $3.7 \mathrm{mg} / \mathrm{dl}$; blood urea nitrogen up to $218 \mathrm{mg} / \mathrm{dl}$ )). Additionally, clinical and physical investigation revealed hepato- and splenomegaly as well as enlarged lymph nodes. Osteomyelitis or other foci of local infections as well as a solid tumor were excluded by radiology or other physical methods (especially with 772 MBq Tc-99 m scintigraphy).

New bone marrow samples (bone marrow biopsy and smear as well as a peripheral blood smear) were taken 1 day after admission to our hospital (10 September 2006). In comparison to the first biopsies in 2004, the bone marrow histology showed the same morphological features (see Fig. 1): (a) pronounced hypercellularity with severe reduction of the age-related adipose tissue, (b) marked granulocytic hyperplasia, normal differentiation of erythro- and megakaryocytopoiesis with slightly reduced number, no blast transformation (c) $1+$ reticulin fibrosis, and (d) enhanced iron deposits in the macrophages. We performed a reverse transcriptase-polymerase chain reaction (RT-PCR) molecular analysis to exclude a classical chronic myeloproliferative disease, which was negative for the major and minor breakpoint cluster region p210 and p190 of the bcr gene and jak-2 (somatic mutation Val617Phe) gene mutation, respectively $[1,2]$. In accordance with the bone marrow histology the bone marrow smear showed pronounced hypercellularity with dominance of segmented granulocytes and without blasts. Cytological signs of hematopoetic dysplasia were not evident. Mainly, segmented or band neutrophilic granulocytes dominated in the peripheral blood smear without enhanced immature cells (promyelocytes, myelocytes or metamyelocytes). Overall, we favored the diagnosis of a chronic neutrophilic leukemia in contrast to the diagnosis in 2004 of a leukemoid reaction. A liver biopsy showed MPOX-positive granulopoiesis intralobular and in the portal tract as well as less frequent erythropoesis and CD61-positive megakaryocytopoiesis indicating advanced extramedullary hematopoiesis.

The patient died due to pulmonary aspergillosis, which was detected 5 days before death by radiological investigations. Autopsy findings confirmed the previous clinical and bioptic findings with extreme hyperplastic and packed hematopoiesis with dominance of the granulopoiesis and extraordinary extramedullary hematopoesis involving liver $(1890 \mathrm{~g})$ and spleen $(980 \mathrm{~g})$ as well as lymph nodes (see Fig. 1g,h). Other hematological disorders such as acute myelogeneous leukemia as well as Hodgkin's or NonHodgkin's lymphoma were excluded by histology and immunohistochemistry postmortem (CD3, CD10, CD20, TDT, MPOX, CD34, CD61, Glycophorin, c-kit, CD68). Additionally, no acute or chronic osteomyelitis was found in the left femur by autopsy.

\section{Discussion}

Based on these findings, we proposed the diagnosis of a myeloproliferative disease and especially the rare form of a chronic neutrophilic leukemia. Comparing our findings with the proposed WHO diagnostic criteria for chronic neutrophilic leukemia [3] there are many arguments supporting the favored diagnosis of CNL: (a) the peripheral blood leukocytes of the patient showed a prolonged leukocytosis with counts over $>=25 \mathrm{~g} / \mathrm{l}$. (b) Every bone marrow biopsy revealed hypercellularity with typical aspects of CNL with segmented neutrophils and bands $>80 \%$ of white cells, immature granulocytes $<10 \%$ of white cells as well as myeloblasts $<1 \%$ in the peripheral blood differential. (c) Molecular analysis detected no Philadelphia chromosome or 

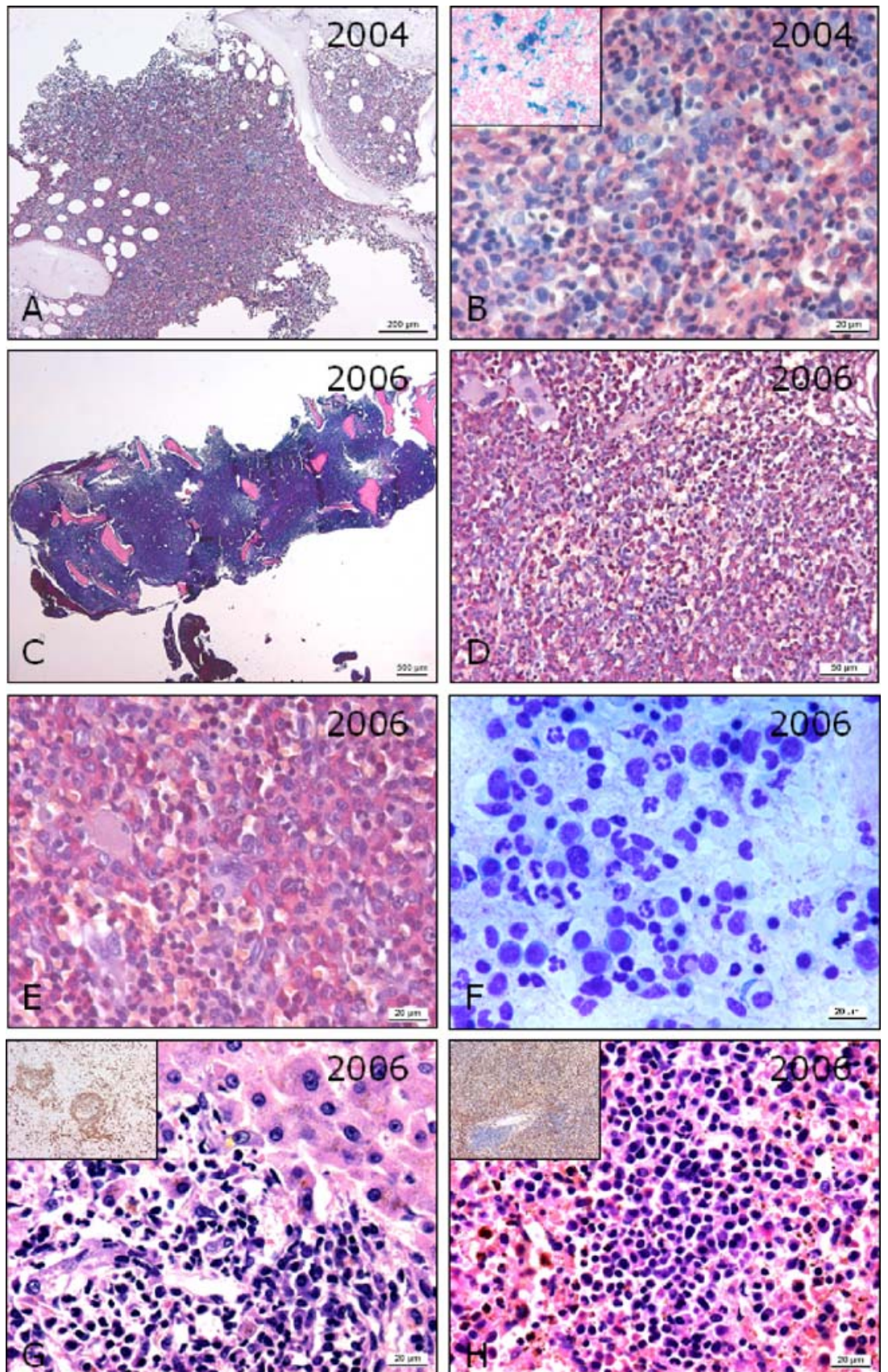

Fig. 1 Consecutive bone marrow biopsies (a-e) and smear (f) taken from 2004 (a and b) to 2006 (c-f) showing imitating "reactive features" of the hematopoesis in the first biopsies (a-b) and giving evidence for the diagnosis of a chronic neutrophilic leukemia later (c-f): striking hematopoetic hypercellularity with reduced age-dependent adipose tissue, dominance of the granulopoesis in contrast to normal erythroand megakaryocytopoesis as well as no blast transformation. Postmor-

$B C R / A B L$ fusion. Additional cytogenetic analysis was not carried out to identify clonality of the granulocytic proliferation or chromosomal alterations which could be observed in about $37 \%$ of cases with CNL [4]. (d) Morphological as well as immunohistochemical analyses of the bone marrow smears or biopsies as well as peripheral blood differential

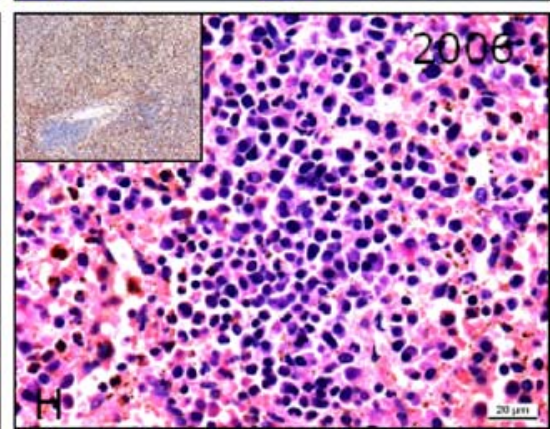

tem analysis revealed extraordinary extramedullary hematopoesis involving liver $(\mathbf{g})$ and spleen $(\mathbf{h})(\mathbf{a} \times 50$ (NASD reaction and $\mathbf{b} \times 400$ (NASD reaction, inset $\mathrm{BB} \times 400)$ as well as $\mathbf{c} \times 12.5(\mathrm{HE}), \mathbf{d} \times 200$ (NASD reaction), e $\times 400$ (NASD reaction) and $\mathbf{f} \times 400$ (MayGrunwald-Giemsa), $\mathbf{g}$ and $\mathbf{h} \times 400$ (HE) and $\times 50$ (MPOX immunohistochemistry) insets, respectively)

excluded the evidence of another myeloproliferative disease (PV, ET, CIMF) or of a myelodysplastic syndrome or myelodysplastic/myeloproliferative disorder. (e) Hepatosplenomegaly was proofed by clinical and autoptic findings. (f) Finally, the WHO criteria for CNL included that no identifiable cause of physiologic neutrophilia was evident. 
Anamnesis of our presented patient revealed a chronic osteomyelitis which could be excluded by radiological and autoptic investigations. The pulmonary aspergillosis was evident in the prefinal phase of the patient in circumstances of progressive extramedullary hematopoesis possibly with functional granulopoesis impairment.

Overall, CNL is a really rare disease. Applying correctly the WHO criteria to cases with suspicious CNL, many of these cases did not meet these criteria [5]. Elliot et. al. presented overall 40 cases being in line with these criteria of CNL [6]: in short, these patients were predominantly older and male (17 female, 23 male; age (years), 66.5 [15-86]) showed a leukocytosis without immature granulocytes or blasts $(\mathrm{Hgb}(\mathrm{g} / \mathrm{dl}), 11.2$ (716.7); PLT (g/l), 136 (7-476); white blood count (g/l), 39.1 (11.3-125.7); polymorphonuclear neutrophils (\%), 87 (6898.5); immature neutrophils, 2 (0-11); Blast (\%), 0), splenomegaly (28/40), cytogenetic abnormalities (8/40 (initial), $+5 / 40$ (during follow-up) $(32.5 \%)$ ) and a short survival time (months: 23.5 (1-94)) as well as different causes of death (intracranial hemorrhage $(n=9)$, progressive disease $(n=5)$, blast transformation $(n=4)$, infection $(n=1)$, regimen-related toxicity post-transplantation $(n=1))$.

The major and sometimes very difficult differential diagnosis of CNL is the leukemoid reaction with leukocyte count greater than $50.00 \mathrm{~g} / 1$ and significant increase in mature neutrophils with a marked left shift. Causes of leukemoid reaction are very heterogeneous ranging from infections, malignancies, drugs, intoxications and severe hemorrhage and acute hemolysis to rare conditions such as alcoholic steatohepatitis indicating that really nothing could be excluded as being responsible for a leukemoid reaction [7]. In our case, the clinical diagnosis of osteomyelitis could be excluded by autopsy and the progressive pulmonary aspergillosis was clinically/radiologically evident only 5 days before death.

To differentiate between leukemoid reaction and CNL as well as chronic myelogenous leukemia (CML) are a mixture of blood, bone marrow and molecular biological investigation has to be applied as follows: (a) peripheral blood and bone marrow, (b) leukocyte alkaline phosphatase, (c) vitamin B12, (d) cytogenetics, (e) immunophenotyping, (f) serum-granulocyte-colony-stimulating factor (G-CSF), and (g) clonality techniques.

Peripheral blood and bone marrow analyses showed a left shift of granulopoiesis with myelocytes and metamyelocytes in case of leukemoid reaction in contrast to a marked neutrophilia without immature cells as well as myelocytes and metamyelocytes in cases of CNL [8]. In contrast, CML displays more immature cells as well as eosinophils and basophils [9]. Additionally, toxic granulations, Doehle bodies, and cytoplasmatic vacuoles could be found in neutrophils of patients with leukemoid reaction
[8]. Leukocyte alkaline phosphatase score is higher in cases of leukemoid reaction and CNL than in cases of CML [4, 10]. Vitamin B12 levels are higher in CNL and CML, but can not be used to differentiate from a leukemoid reaction [7]. Cytogenetics analyses are negative in cases of leukemoid reaction, whereas in $37 \%$ of $\mathrm{CNL}$ cases abnormalities can be found (especially in chromosome 20) [4]. Interestingly, approximately $20 \%$ of these patients with CNL had chromosomal abnormalities already at baseline or presentation, while about $10-15 \%$ developed over time [6]. The hallmark of CML is the presence of Philadelphia 1 chromosome in the karyotype or the detection of $t(9 ; 22)$ translocation, which is not present in $10 \%$ of atypical myeloproliferative disorders [11]. Additionally, Philadelphia chromosome negative CML with p230 Bcr-abl gene product displays distinct neutrophilia [9]. Immunophenotyping is useful to exclude blast transformation and therefore indicates a true malignant hematological disorder. Serum-G-CSF is low in cases of CNL and CML in contrast to higher levels in cases of a leukemoid reaction which is normally not available in a standard laboratory [7]. Finally clonality techniques such as the progenitor cell assays [12], the $\mathrm{X}$ inactivation patterns [13] and reduced susceptibility of malignant neutrophils to apoptosis [14] are useful to identify clonality in cases of $\mathrm{CNL} / \mathrm{CML}$ and not of leukemoid reaction which is reserved for specialized laboratories. Additionally, microbiological as well as physical examinations such as CT or NMR could be helpful to detect a focus of infection or a malignant tumor.

Relating to our case, we were not able to carry out investigations such as leukocyte alkaline phosphatase, cytogenetics, serum-G-CSF and clonality techniques. Nevertheless, the rest of the above mentioned analyses give evidence for a chronic neutrophilic leukemia: vitamin B12 was highly elevated and a marked neutrophilia without dysplasia and immature cells was seen in peripheral blood and bone marrow specimen. Immunohistochemistry could exclude a blast transformation. Additionally, the major and minor breakpoint cluster region p210 and p190 of the $b c r$ gene and jak-2 (somatic mutation Val617Phe) as well as blast excess were excluded by RT-PCR and immunohistochemistry. Although a neutrophilic variant of a CML with p230 Bcr-abl gene product could not be excluded by our molecular analysis, the presented morphological phenotype of our case is not constituent with an atypical CML or a Ph1/bcr+-CML but with the rare diagnosis CNL [9].

Taken together not as a single criterion, but as a sometimes time-dependent consecutive combination of these "standard laboratory" criteria (such as bone marrow smear and biopsy as well as peripheral blood analysis) can be helpful in the diagnostic challenge for discriminating between reactive hematopoiesis and rare chronic neutrophilic leukemia. 


\section{References}

1. Gabert J, Beillard E, van dV V et al (2003) Standardization and quality control studies of 'real-time' quantitative reverse transcriptase polymerase chain reaction of fusion gene transcripts for residual disease detection in leukemia - a Europe Against Cancer program. Leukemia 17(12):2318-2357

2. Kralovics R, Passamonti F, Buser AS et al (2005) A gain-offunction mutation of JAK2 in myeloproliferative disorders. N Engl J Med 352(17):1779-1790

3. World Health Organization (2001) Classification of tomours: Pathology and genetics of tumours amd lymphoid tissue. IARC, Lyon, France

4. Reilly JT (2002) Chronic neutrophilic leukaemia: a distinct clinical entity? Br J Haematol 116(1):10-18

5. Elliott MA (2004) Chronic neutrophilic leukemia: a contemporary review. Curr Hematol Rep 3(3):210-217

6. Elliott MA, Hanson CA, Dewald GW, Smoley SA, Lasho TL, Tefferi A (2005) WHO-defined chronic neutrophilic leukemia: a long-term analysis of 12 cases and a critical review of the literature. Leukemia 19(2):313-317

7. Sakka V, Tsiodras S, Giamarellos-Bourboulis EJ, Giamarellou H (2006) An update on the etiology and diagnostic evaluation of a leukemoid reaction. Eur J Intern Med 17(6):394-398
8. Dinauer MC, Coates TD (2005) Disorders of phagoycyte function and number. In: Hoffmann R, Benz EJ, Shattil SJ, Furie B, Cohen HJ, Silberstein LE, McGlave P (eds) Hematology: basic principles and practice. Philadelphia, Churchill Livingstone, pp 737-762

9. Kvasnicka HM (2002) Diagnostic spectrum of Philadelphiachromosome negative leukemic disorders. Pathologe 23(6): $448-456$

10. Nimieri HS, Makoni SN, Madziwa FH, Nemiary DS (2003) Leukemoid reaction response to chemotherapy and radiotherapy in a patient with cervical carcinoma. Ann Hematol 82(5):316-317

11. Costello R, Sainty D, Lafage-Pochitaloff M, Gabert J (1997) Clinical and biological aspects of Philadelphia-negative/BCRnegative chronic myeloid leukemia. Leuk Lymphoma 25(3-4): 225-232

12. Yanagisawa K, Ohminami H, Sato $M$ et al (1998) Neoplastic involvement of granulocytic lineage, not granulocytic-monocytic, monocytic, or erythrocytic lineage, in a patient with chronic neutrophilic leukemia. Am J Hematol 57(3):221-224

13. Bohm J, Kock S, Schaefer HE, Fisch P (2003) Evidence of clonality in chronic neutrophilic leukaemia. J Clin Pathol 56(4): 292-295

14. Kobayashi S, Yamashita K, Takeoka T et al (2002) Calpainmediated X-linked inhibitor of apoptosis degradation in neutrophil apoptosis and its impairment in chronic neutrophilic leukemia. J Biol Chem 277(37):33968-33977 